\title{
Vaccines against Toxoplasma gondii
}

\author{
Status, challenges and future directions
}

Qi Liu, ${ }^{1}$ Lachhman Das Singla ${ }^{2}$ and Huaiyu Zhou ${ }^{1, *}$

${ }^{1}$ Department of Parasitology; Shandong University School of Medicine; Jinan, Shandong, China; ${ }^{2}$ Department of Veterinary Parasitology; College of Veterinary

Science; Guru Angad Dev Veterinary and Animal Sciences University; Ludhiana, India

$T$ Jxoplasma gondii is a ubiquitous protozoan parasite that can infect a wide range of animals including humans. This single known species in the genus Toxoplasma is considered as one of the most successful eukaryotic pathogens which is of major medical and veterinary importance. Effective vaccines may contribute toward preventing and controlling the spread of toxoplasmosis. The present communication addresses the current status of development of vaccines against $T$. gondii. Further discussion is made on the difficulties along with challenges, such as vaccine construct, mode of vaccine administration and standardization of immunization evaluation. Finally suggestions are made on possible directions for future research on the development of vaccines against $T$. gondii.

\section{Introduction}

It has been more than 100 years since Toxoplasma gondii was first described in the tissues of an african rodent, Ctenodactylus gundi by Nicolle and Manceaux. ${ }^{1} T$. gondii has a complex life cycle consisting of a sexual cycle in its feline definitive hosts and an asexual cycle in its intermediate hosts. ${ }^{1}$ There are three functionally distinct pathogenic forms, sporozoites (in oocysts), tachyzoites and bradyzoites (in tissue cysts). Intermediate hosts, including humans, can be exposed to and infected with $T$. gondii via tissue-cyst ingestion (carnivorism), oocyst ingestion (fecaloral route) and congenital (transplacental) infection. As an obligate intracellular apicomplexan protozoan, T. gondii can infect virtually all warm-blooded animals, including mammals and birds. The parasite is ubiquitous throughout the world and is estimated to infect approximately half of the world's population. Infection can result in encephalitis in immunocompormised hosts, chorioretinitis in immunocompetent hosts or serious congenital disease in developing fetus if pregnant women become infected for the first time during pregnancy. ${ }^{2}$ In addition, infection in domestic animals is a threat to public health from food-borne outbreaks and causes a great economic loss as it may lead to abortion, stillbirth and neonatal loss. ${ }^{3}$ Therefore, toxoplasmosis is of great medical and veterinary importance. Treatment of this disease is difficult since the available drugs have severe side effects and reactivation may occur at any time. ${ }^{2,4}$ Under the present scenario, therefore, developing an effective vaccine against $T$. gondii infection is the need of the hour.

At present, only one commercial vaccine "Toxovax" based on live attenuated S48 strain has been licensed for use to avoid congenital infection in ewes. ${ }^{5}$ However, this vaccine is expensive, causes adverse effects, and has a short shelf-life. Furthermore, this vaccine may revert to a pathogenic strain and therefore it is not suitable for human use. ${ }^{6}$ There is currently no licensed vaccine available for humans.

In order to address this problem, numerous vaccination trials against toxoplasmosis have been performed for their immunological effects in animal models, including inactivated and attenuated vaccine, subunit vaccine, genetically engineered vaccine and DNA vaccine. The 
results have shown that it may be possible to develop an effective vaccine against toxoplasmosis.

\section{Current Developments and Challenges in the Production of Vaccines against $T$. gondii}

In recent years, tremendous progress has been made to search for a vaccine that can help prevent toxoplasmosis, but there is still a little to offer against this ubiquitous pathogen. Problems encountered in the research for the development of vaccine against $T$. gondii have been discussed below.

Inactivated, killed and crude antigen vaccines of $T$. gondii, can probably not be sufficiently efficacious in any of the infection models used. ${ }^{6}$ In contrast, liveattenuated vaccines are capable of mobilizing a MHC class I-restricted CD8+ T-cell response, which has been considered the major response required to clear intracellular infections caused by parasites, and generally elicit similar protection to natural primary infection. ${ }^{6}$ However, the immunogenicity of live vaccines can be significantly influenced by their degrees of attenuation, and the application of these vaccines has been relatively restricted without a reasonable shelf-life. There are also theoretical safety concerns as attenuated vaccines carry the risk of reverting to a pathogenic strain. ${ }^{7}$ Subunit vaccines tend to lack immunogenicity and therefore require the use of appropriate adjuvants to enhance their potency. Additionally, subunit vaccines could cause an allergic reaction in the individuals since the protein or the protein subunit expressed within another organism such as bacteria or yeast. DNA vaccines have some distinct advantages compared with traditional vaccines in that they can elicit cellular and humoral immune responses against toxoplasmosis. The $\mathrm{CD} 8^{+} \mathrm{T}$ cell response induced by DNA vaccine is the major mediator of immunity against $T$. gondii infection which makes it a promising approach against intracellular parasites. ${ }^{6}$ However, it has been recognized that DNA vaccines generate only weak immune responses when used in higher primates and humans. Various efforts have been made to improve DNA vaccine potency, for example, using various DNA delivery systems such as electroporation and a gene gun approach, modifying the microenvironment of the vaccination site via co-expression of cytokines, chemokines, co-stimulatory molecules as molecular adjuvants, optimizing codon to maximize protein expression and using heterologous prime-boost strategy. ${ }^{6-8}$

\section{Factors Restricting the Development of $T$. gondii Vaccine}

Lack of efficacious protective antigen candidates. Due to complex life cycle involving multiple hosts with diversity of protein forms to express and variety of invasion pathways, $T$. gondii exhibits a very high degree of antigenic polymorphism and variability. Although several candidate antigens have been identified in the past few years, the ones capable of inducing a strong and long-lasting protective immunity are limited. Therefore, selection of target antigens is a crucial step to design effective vaccines and work has focused on identifying antigens essential for parasite survival in the host or that are recognized by protective antibodies or T cells. ${ }^{9}$ In addition, accumulating evidences indicate that vaccination with stage-specific antigens leads to stage-limited protection against $T$. gondii. ${ }^{10}$ Thus, it is essential to develop multiantigenic vaccines in connection with different growth stages in the life cycle in order to conquer the deficiency of using single antigen as vaccine candidate.

To date, we still have to evaluate vaccine efficacy without a clear knowledge of how it works. This often leads to wastage of the limited funds available for $T$. gondii vaccine study. Additionally, a very common model employed in vaccine development is the mouse, yet its immune system is known to differ quite radically from humans. ${ }^{11}$ While some vaccines have shown fascinating results in animal models, it is difficult to extend the findings to predict the outcome of using such a vaccine approach in humans.

Lack of detailed understanding of pathogenic, immune and host cell invasion mechanisms. Due to more complicated biological characteristics, a variety of invasion pathways and mechanisms to evade host immune responses, development of an effective vaccine against $T$. gondii appears to be more difficult than general bacterial or viral infections. As $T$. gondii is an obligate intracellular parasite, cellular immunity has been considered the major response to eliminate the parasite within the host, yet humoral immunity also plays an important role in shaping the immune responses..$^{12}$ However, immunity to $T$. gondii is complex, and involves many facets of the immune system, and our understanding of the immune mechanism as well as pathogenesis and host cell invasion mechanisms remain incomplete. While these are lacking, vaccine development is considerably more problematic, and is likely to lose direction, and also cannot tailor ideal vaccine against $T$. gondii. For example, cytokines secreted in the immune response to $T$. gondii include upregulated factors (IFN $\gamma$, IL-2, TNF $\alpha$, IL-1, IL-7, IL-12, IL-15) and downregulated factors (IL-4, IL-6, IL-10). Only when certain balance between high expression of upregulated ones and low expression of downregulated ones is achieved, can T. gondii not evade the host immune response. ${ }^{12,13}$ Furthermore, the protective immunity exhibited in animal models may differ considerably from humans. Thus, the current researches are filled with blindness.

Insufficient advanced research techniques and theories of immunology or vaccinology. Many genetically engineered recombinant proteins extracted from inclusion bodies are mostly denatured ones which lack of necessary functional groups. It brings disillusionary consequence that while the immune antibody titer is high, the immune protection is very low. Moreover, there is no selectivity when referring to the advanced technologies, especially in the research of genetically engineered vaccines. Many researchers still hold the partial opinion that genetically engineered recombinant vaccine (especially recombinant DNA plasmid) is the only direction of vaccine development. Additionally each vaccine candidate has to be investigated in animal models.

Can DNA vaccines fulfill their promise? DNA vaccines have opened up a promising approach against toxoplasmosis as they showed more prominent 
advantages over traditional vaccines in terms of flexibility, rapid manufacture, low cost and ability to induce cellular and humoral immunity. Nevertheless, there are ongoing challenges calling for further research advances for development of DNA vaccines against $T$. gondii.

Biosafety of DNA vaccines. The core issue for DNA vaccine is safety. As an exogenous material, DNA vaccines have the possibility to integrate partial or complete plasmid sequences into the host genome, causing insertion mutations with activation of oncogenes or inactivation of tumor suppressor genes. ${ }^{13}$ The situation may be much worse if plasmid DNA spread to the reproductive cells and integration occurs. Fortunately, experimental data have shown that the rate of plasmid integration is negligible and lower than the spontaneous rate of mutation in mammalian genomes. ${ }^{14}$ In addition, to date a large sample of PCR detection has not observed plasmid integration into the host genome. It could be argued, therefore, DNA vaccines are considered to be safe for now.

Immune tolerance and allergy problems of DNA vaccines. Another safety issue includes the possibility that DNA vaccines could elicit immune tolerance and allergic reaction. Fortunately, data have revealed that immune tolerance rarely occurs except for newborns administered with DNA vaccines. ${ }^{15}$ Additionally, DNA vaccines could increase the production of anti-DNA autoantibodies but did not increase disease severity in lupusprone animals nor induce autoimmunity in healthy animals. ${ }^{15}$ The presence of antiDNA autoantibodies is a characteristic feature of systemic autoimmune diseases such as systemic lupus erythematosus (SLE). However, early studies showed that anti-DNA autoantibodies elicited after DNA inoculation could not trigger autoimmune disorders, which appears to be due to the micro skeleton of plasmid, little dose of injection and weak immunogenicity of DNA. ${ }^{16}$

The efficacy of DNA vaccines. Suboptimal immunogenicity is an ongoing barrier for DNA vaccines. The higher is the group of the experimental animal species, the weaker are the immune responses to DNA vaccines generated. Genetic differences between species may be one of the reasons for the detailed mechanisms supposed to be explored. It is in urgent need to address the poor immunogenicity when used in higher primates and humans for the application of DNA vaccines.

Further research advances are required to better understand DNA vaccine action such as the expression and regulation of antigen genes after injecting the combined plasmid into the body. It is encouraging that several DNA vaccines have already been licensed for veterinary applications including West Nile virus in horses, infectious hematopoietic necrosis factor disease in salmon and melanoma in dogs. ${ }^{17}$ Simultaneously, DNA vaccines for AIDS, malaria, hepatitis $B$ and some cancers are now in clinical trials and could be available in the near future. ${ }^{17}$ All these facts reinforce the view that with recent technology breakthroughs, effective human DNA vaccines against $T$. gondii may finally deliver on their potential.

\section{Directions for Future Research}

Future studies should focus on devising effective vaccines, to select target antigens and then present them to the immune system via an optimal delivery strategy to stimulate appropriate protective immunity. The major challenge still lies in the lack of efficacious vaccine antigen candidates. Dramatically, antigen selection methods have altered in the postgenomic era, with the development of bioinformatics, genomics and proteomics, which may seek more novel and effective vaccine candidates. Moreover vaccines against toxoplasmosis should have different antigens expressed in all stages of the parasite associated with adequate adjuvant and appropriate delivery strategy. There is an increasing awareness that selection of the vaccine candidates, selection of the adjuvants and choice of delivery methods is of the same importance. As an indispensable part of vaccine, adjuvant plays a critical role in enhancing immunogenicity of weaker antigen and activating an appropriate immune response safely without multiple dose regimens. Many adjuvants have been licensed and used for years although the mechanism of adjuvant action is still unclear. ${ }^{18}$ Further research should consider the mode of action for traditional adjuvants and the development of new adjuvants based on pattern recognition receptors (PRRs), such as the TLRs, NOD-like receptors and C-type lectin receptors. ${ }^{19}$

Future studies should address the issues of standardizing immunization protocol and evaluation criteria. Regardless of the vaccine constructs, many parameters such as the parasite strain, the dose of inoculum, the inoculation route and the mouse strain, might hinder the assessment of protective immunity. ${ }^{20}$ Our previous works in T. gondii protein vaccine or DNA vaccine show that the major limitation of the lack of a full evaluation of vaccines is probably due to an inappropriate challenge protocol. ${ }^{21,22}$ As one review reveals, vaccine studies should focus on standardizing the immunization protocol and comparing the immunogenicity of a fixed antigen delivered with different vectors and adjuvants in order to solve the complex puzzle of the $T$. gondii vaccine. ${ }^{20}$

Future studies should not ignore the mechanisms of pathogenic, immune and host cell invasion associated with $T$. gondii as well as vaccine action against toxoplasmosis. With the increasing progress of molecular biology and immunology and delivery system techniques, molecular vaccines against $T$. gondii is bound to be a major breakthrough. Particularly, the application of genomics and proteomics in $T$. gondii not only clarifies the profiles of the parasite and its protein function, but also provide some novel and effective broad-spectrum targets for drugs and vaccines against $T$. gondii. ${ }^{23}$

In summary, $T$. gondii is a highly successful parasitic organism that is able to infect and live within a very wide range of different animal hosts. In most immunocompetent individuals, infection with $T$. gondii results in development of protective immune response against the disease. In addition, chronically infected women can exhibit a degree of immunity normally sufficient to protect their fetus from congenital infection even if re-exposed during pregnancy. ${ }^{4,10}$ These evidences therefore would suggest that vaccine against toxoplasmosis has a high likelihood of success. Although there is currently no licensed $T$. gondii vaccine available for human, we must 
remain optimistic that it will be possible to develop an effective and safe vaccine against toxoplasmosis for animals and humans.

\section{Acknowledgements}

The authors would like to acknowledge the support of the Outstanding Young Scientists Research Award Project of Shandong, China (No. BS2009SW008) and the Science and Technology Development Program of Shandong, China (No. 2006GG3202045). The authors declare no conflict of interest.

\section{References}

1. Dubey JP. History of the discovery of the life cycle of Toxoplasma gondii. Int J Parasitol 2009; 39:87782; PMID:19630138; http://dx.doi.org/10.1016/j. ijpara.2009.01.005.

2. Montoya JG, Liesenfeld O. Toxoplasmosis. Lancet 2004; 363:1965-76; PMID:15194258; http://dx.doi. org/10.1016/S0140-6736(04)16412-X.

3. Jones JL, Dubey JP. Foodborne Toxoplasmosis. Clin Infect Dis 2012; PMID:22618566; http://dx.doi. org/10.1093/cid/cis508.

4. Serranti D, Buonsenso D, Valentini P. Congenital toxoplasmosis treatment. Eur Rev Med Pharmacol Sci 2011; 15:193-8; PMID:21434486.

5. Buxton D, Innes EA. A commercial vaccine for ovine toxoplasmosis. Parasitology 1995; 110:116; PMID:7784124; http://dx.doi.org/10.1017/ S003118200000144X
6. Kur J, Holec-Gasior L, Hiszczy ska-Sawicka E. Current status of toxoplasmosis vaccine development. Expert Rev Vaccines 2009; 8:791808; PMID:19485758; http://dx.doi.org/10.1586/ erv.09.27.

7. Garcia JL. Vaccination concepts against Toxoplasma gondii. Expert Rev Vaccines 2009; 8:215-25; PMID:19196201; http://dx.doi. org/10.1586/14760584.8.2.215.

8. Saade F, Petrovsky N. Technologies for enhanced efficacy of DNA vaccines. Expert Rev Vaccines 2012; 11:189-209; PMID:22309668; http://dx.doi. org/10.1586/erv.11.188.

9. Innes EA, Vermeulen AN. Vaccination as a control strategy against the coccidial parasites Eimeria, Toxoplasma and Neospora. Parasitology 2006; 133:145-68; PMID:17274844; http://dx.doi. org/10.1017/S0031182006001855.

10. Alexander J, Jebbari H, Bluethmann H, Satoskar A, Roberts CW. Immunological control of Toxoplasma gondii and appropriate vaccine design. Curr Top Microbiol Immunol 1996; 219:183-95; PMID:8791700.

11. Mestas J, Hughes CC. Of mice and not men: differences between mouse and human immunology. J Immunol 2004; 172:2731-8; PMID:14978070.

12. Denkers EY, Gazzinelli RT. Regulation and function of T-cell-mediated immunity during Toxoplasma gondii infection. Clin Microbiol Rev 1998; 11:56988; PMID:9767056.

13. Lang C, Gross U, Lüder CG. Subversion of innate and adaptive immune responses by Toxoplasma gondii. Parasitol Res 2007; 100:191-203; PMID:17024357; http://dx.doi.org/10.1007/s00436-006-0306-9.

14. Faurez F, Dory D, Le Moigne V, Gravier R, Jestin A. Biosafety of DNA vaccines: New generation of DNA vectors and current knowledge on the fate of plasmids after injection. Vaccine 2010; 28:388895; PMID:20371391; http://dx.doi.org/10.1016/j. vaccine.2010.03.040.
15. Klinman DM, Takeshita F, Kamstrup S, Takeshit $\mathrm{S}$, Ishii $\mathrm{K}$, Ichino $\mathrm{M}$, et al. DNA vaccines: capacity to induce auto-immunity and tolerance. Dev Biol (Basel) 2000; 104:45-51; PMID:11713823.

16. Lai WC, Bennett M. DNA vaccines. Crit Rev Immunol 1998; 18:449-84; PMID:9784969.

17. Liu MA. DNA vaccines: an historical perspective and view to the future. Immunol Rev 2011; 239:62-84; PMID:21198665; http://dx.doi.org/10.1111/j.1600065X.2010.00980.x.

18. Pulendran B, Ahmed R. Immunological mechanisms of vaccination. Nat Immunol 2011; 12:509 17; PMID:21739679; http://dx.doi.org/10.1038/ ni.2039.

19. Higgins SC, Mills KH. TLR, NLR Agonists and Other Immune Modulators as Infectious Disease Vaccine Adjuvants. Curr Infect Dis Rep 2010; 12:4 12; PMID:21308494; http://dx.doi.org/10.1007/ s11908-009-0080-9.

20. Jongert E, Roberts CW, Gargano N, Förster-Waldl E, Petersen E. Vaccines against Toxoplasma gondii: challenges and opportunities. Mem Inst Oswaldo Cruz 2009; 104:252-66; PMID:19430651; http://dx.doi. org/10.1590/S0074-02762009000200019.

21. Zhou H, Gu Q, Zhao Q, Zhang J, Cong H, Li $\mathrm{Y}$, et al. Toxoplasma gondii: expression and characterization of a recombinant protein containing SAG1 and GRA2 in Pichia pastoris. Parasitol Res 2007; 100:829-35; PMID:17058109; http://dx.doi. org/10.1007/s00436-006-0341-6.

22. Zhou H, Min J, Zhao Q, Gu Q, Cong H, Li Y, et al. Protective immune response against Toxoplasma gondii elicited by a recombinant DNA vaccine with a novel genetic adjuvant. Vaccine 2012; 30:1800-6; PMID:22240340; http://dx.doi.org/10.1016/j.vaccine.2012.01.004.

23. Weiss LM, Fiser A, Angeletti RH, Kim K. Toxoplasme gondii proteomics. Expert Rev Proteomics 2009; 6:303-13; PMID:19489701; http://dx.doi. org/10.1586/epr.09.16. 